\title{
Complementarity of Neutron and Ultrahigh Resolution Synchrotron X-Ray Protein Crystallography Studies: Results with Concanavalin A at Cryo and Room Temperature
}

\author{
J.R. Helliwell* ${ }^{*}$ H.J. Price, A. Deacon ${ }^{\dagger}$, J. Raftery \\ AND J. HABASH \\ Department of Chemistry, University of Manchester, M13 9PL, UK
}

\begin{abstract}
The complementarity of synchrotron derived ultrahigh resolution X-ray and neutron protein crystallography is explored via an ensemble of plant lectin concanavalin A crystal structures. Thus a resume of a study of a cryo $0.94 \AA$ and a neutron ( $+\mathrm{X}$-ray) protein crystal $2.4 \AA$ structure at room temperature is made and these are then compared in their efficiency to determine the positions of the bound solvent atoms i.e. as hydrogens or deuteriums. First results are also presented of comparisons of two ultrahigh resolution protein crystal structures, the $0.94 \AA$ and a new $0.92 \AA$ structure. Thus the variability of the two cryo structures, at very fine detail, is described; this variability is in the multiple occupancies of side chains. Overall, one can see that a "complete" structure definition, with today's experimental capabilities, is possible and can include structure ensemble variations.
\end{abstract}

PACS numbers: 87.64.- -t, 87.15.--v, 61.12.--q

\section{Introduction}

In protein crystallography today the definition of a protein structure can be in a complete form. Thus other structural scientists can have access, in quite

*corresponding author; e-mail: j.r.helliwell@dl.ac.uk; john.helliwell@man.ac.uk; current address: CLRC Director of SR Science, Daresbury Laboratory, Warrington WA4 4AD, UK

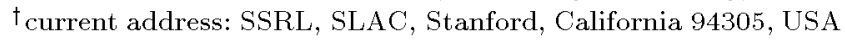


a number of cases today, to a structure at its most detailed, i.e. ultrahigh resolution. This generally means harnessing cryo-temperature. I personally do wonder however about the variation of structure from physiological temperature to cryo-temperature, not least as this crosses the well known glass transition [1] at $\approx 180 \mathrm{~K}$, and where function is lost. Moreover, hydrogen atoms details can still be missing in an ultrahigh resolution protein crystal structure due to part mobility of these atoms. Neutron protein crystallography allows much improved definition of hydrogen positions, as deuteriums, since the neutron scattering factor for deuterium matches carbon, nitrogen, and oxygen. Hence the neutron approach tolerates the partial mobility problem better than X-rays, and even $2.5 \AA$ resolution neutron structures offer deuterium atom position details. (Obviously fully mobile atoms cannot be determined by any diffraction technique.) The bound water deuteriums are a whole class of atoms that are generally missing from X-ray protein crystal structures. Neutrons are also non-destructive as an experimental probe so radiation damage is not a limitation as it really can be with X-rays. We find ourselves defining the complete protein structure then as needing to be relevant to physiological temperature but as detailed as possible. Ideally, one can foresee the need for an X-ray structure at both cryo and room temperature and a neutron structure of the deuterated form, which can be at room temperature. Thus, at least with one protein structure, concanavalin A, our Structural Chemistry Laboratory in Manchester has steadily provided an ensemble of structures to "define" this protein. Concanavalin A is of interest on two principal counts. Firstly, biologically, as a saccharide binding protein it is implicated in cell to cell cross linking. Secondly, biophysically, it is extensively used as a test system for characterisation of ligand binding and molecular recognition studies. It is the most extensively studied of the plant lectins. Careful structural definition is therefore worthwhile.

\section{Experiments}

Highly resolved crystal structures of protein molecules can be obtained now using X-ray crystallographic data measured at synchrotron radiation sources with high performance detectors and cryoprotected samples. Details of the mobility of the atoms is also available from their "thermal parameters" in the refined molecular model. In this way, we have now studied the protein to $0.94 \AA$ resolution [2]. The ease of exchange of particular protons for deuterium has then been studied using neutron Laue diffraction data measured at the ILL in Grenoble [3]. In addition we have determined the $\mathrm{D}_{2} \mathrm{O}$ bound solvent structure of concanavalin $\mathrm{A}$ using a combined $\mathrm{Cu} K$ alpha $\mathrm{X}$-ray refinement at $1.8 \AA$ and neutron at $2.4 \AA$ refinement approach, and then compared the results with the bound solvent in the $0.94 \AA$ SR X-ray structure [4], Table I. Finally, most recently, we have determined another ultrahigh resolution concanavalin A (the $\mathrm{Mn}, \mathrm{Ca}$ saccharide free form) at $0.92 \AA$ resolution [5]. 


\section{Results}

Many more bound water molecule deuteriums can be located via neutron protein crystallography (" $n+X$ " approach) rather than the ultrahigh resolution X-ray approach alone. However the latter does deliver a few very detailed water molecule hydrogen atoms (see Table I), as well as its highly detailed overall structure. The two approaches are thus complementary.

TABLE I

Bound water comparison: $2.4 \AA$ resolution neutron (+ X-ray) versus $0.94 \AA$ SR X-ray cryo-temperature (from Ref. [4]).

\begin{tabular}{c|c|c|c|c|c|c|c}
\hline \hline $\begin{array}{c}\text { Temperature } \\
{[\mathrm{K}]}\end{array}$ & Technique & Total waters & $\begin{array}{c}\text { Common bound } \\
\text { waters to each }\end{array}$ & $\mathrm{D}_{2} \mathrm{O}$ & $\mathrm{DO}$ & $\mathrm{H}_{2} \mathrm{O}$ & $\mathrm{HO}$ \\
\hline 293 & "n+X" & 148 & 88 & 62 & 20 & & \\
100 & SR X-ray & 319 & & & & 12 & 35
\end{tabular}

TABLE II

Protein structure variations between room and cryo temperatures (from Ref. [5] and to be published) seen in terms of the number of multiple occupancy side chain cases (the remaining parts of the whole protein structure remaining essentially the same, within their respective protein structure precisions).

\begin{tabular}{|c|c|c|c|c|}
\hline Case & & & Common & Total \\
\hline (i) cryo to cryo & $\begin{array}{c}0.94 \AA \text { "native" } \\
\text { concanavalin A } 25\end{array}$ & $\begin{array}{c}0.92 \AA \mathrm{Mn}, \mathrm{Ca} \\
\text { concanavalin A } 39\end{array}$ & 22 & 42 \\
\hline $\begin{array}{l}\text { (ii) room } \\
\text { versus room } \\
\text { temperature }\end{array}$ & $\begin{array}{c}1.7 \AA \mathrm{Mn}, \mathrm{Ca} \\
\text { concanavalin A } 5\end{array}$ & $\begin{array}{c}1.6 \AA \mathrm{Co}, \mathrm{Ca} \\
\text { concanavalin A } 5\end{array}$ & 4 & 6 \\
\hline $\begin{array}{l}\text { (iii) room } \\
\text { versus cryo }\end{array}$ & $\begin{array}{c}1.7 \AA \mathrm{Mn}, \mathrm{Ca} \\
\text { concanavalin A } 5\end{array}$ & $\begin{array}{c}0.92 \AA \mathrm{Mn}, \mathrm{Ca} \\
\text { concanavalin A } 39\end{array}$ & 3 & 42 \\
\hline
\end{tabular}

There are differences between, and within, these cryo and room temperature protein structures; they represent an ensemble of experimental refined structures of concanavalin A [5]. There are variations apparent in structure between the cryo and room temperatures, and within a temperature (see Table II).

\section{Further developments}

(i) Room temperature protein structure definition is important because this is closer to physiological temperature, but how to improve the resolution obtainable with X-rays? The study and characterisation of protein crystal perfection has 
revealed that the mosaicity of crystals at room temperature is even at the arc second value. Hence the strict collimation of undulator X-rays offers an improved measurement physics of ultrafine-phi-slicing for data collection. Hence, by working with lower SR intensities, i.e. unfocussed, but near-plane wave, the signal to background can be enhanced, the weak data measured more accurately and the resolution limit perhaps extended. Work to this end is underway principally by Snell, Borgstahl and Bellamy using SSRL, Stanford. Moreover, microgravity provides a medium for convection free growth of large crystals for which improved perfection can be achieved [6].

(ii) To widen the applicability of neutron protein crystallography, the extension of neutron data collection methods to encompass yet larger unit cells is important. In Manchester, using the ILL LADI in Grenoble, we have probed this technical frontier. We have shown we can currently record neutron data, using large crystals (approximately $3 \mathrm{~mm}$ in size), on the cubic concanavalin A with bound glucoside (space group $[213, a=168 \AA$ ) to $\approx 3.5 \AA$ [7]. In order to reach $3.0 \AA$ or better resolution, improved measuring techniques are needed. One way, being explored by Blakeley and Myles, is to freeze the protein. This is challenging for large crystals whilst preserving their perfection (keeping the mosaicity to a reasonable value for data collection) but a cryo neutron data set on the standard concanavalin A crystal form has been achieved recently at the ILL LADI instrument in Grenoble. In another approach, time-of-flight Laue diffraction is an attractive method as the signal to noise can be improved, but is yet to be brought to fruition [8]. The proton synchrotron spallation "short pulse" type neutron source (SNS) such as ISIS @ $160 \mathrm{~kW}$, especially the proposed cold target station 2, and then the upcoming USA's SNS (@2 MW) and the Japanese spallation neutron project, will each allow the time-of-flight Laue approach to be utilised and at an increasing source power level. In the limit of the proposed ESS @ 5MW, with the short pulse target station, a factor of up to approximately 30 gain in "signal to background" capability would be available over ISIS and 10× ILL LADI [9]. Thus, major strides can be made to address two fundamental limits of neutron protein crystallography at present. Firstly, smaller protein crystals could be harnessed e.g. down to $0.5 \mathrm{~mm}$ on edge for a "typical" 25,000 molecular weight protein (in the crystal asymmetric unit) [7]. Secondly, larger molecular weight proteins (in the crystal asymmetric unit) could be studied where large crystals can be grown (e.g. $3 \mathrm{~mm}$ on edge is a "typical" maximum [7]). Since the molecular weight histogram for e.g. the yeast genome peaks around 30,000 molecular weight, and many proteins make oligomers of single subunits, a larger molecular weight capability for neutron protein crystallography is vital. ESS can provide this. Overall ESS can allow then the detailed structural dissection of enzymes whose mechanisms need definition of key hydrogens (as deuteriums). Furthermore biological macromolecule ligand interactions involve water displacement and this is an even bigger set of targets for neutron structural study, including proteins and nucleic acids. 


\section{Concluding remarks}

Given the relative ease and wide availability of protein crystallography data collection today, especially using SR X-rays, ensembles of X-ray structures of a protein can be obtained. Neutron protein structures are rarer to come by. However, the synergy between the SR Laue development and its application, via software package transfer, for neutron protein data collection and analysis has meant that strides have been made in recent years to speed up the delivery of neutron results (for details see Refs. [4] and [7]). The efficacy of neutrons as a non-destructive probe is also important. Moreover, the extra mobility of bound waters at room temperature makes their study via $\mathrm{X}$-rays yet more challenging, since $\mathrm{X}$-rays cause radiation damage at such temperatures, and which further increases the utility and contribution of the neutron approach.

In terms of variations in protein structure, between cryo and room temperature, more studies of other protein systems are of course needed to confirm the generality of these results.

\section{Acknowledgments}

The work described in this article was conducted at CHESS, Cornell for the SR X-ray concanavalin A structures at 0.94 and $0.92 \AA$ resolution. The neutron protein crystallography experiments were conducted at the Institut Laue Langevin Grenoble France "LADI" instrument. Both Facilities are thanked for their support, conducted under peer reviewed beam time awards to J.R.H. The University of Manchester is thanked for general support. The EPSRC is thanked for a Ph.D. studentship awarded to Ashley Deacon. The UK Israel Fund award to J.R.H. and A.J. Kalb (Gilboa) of the Weizmann Institute supported H.J. Price with a Ph.D. studentship. The BBSRC provided a research grant to J.R.H. and W.N. Hunter for the Manchester Structural Chemistry "Biomolecular computer suite", later enhanced by a research grant award from the Wellcome Trust to J.R.H., which also provided salary support to Dr J. Habash. Dr Madeleine Helliwell is thanked for her critical reading of the manuscript.

Finally the close collaboration with Professor Jacek Grochowski concerned with the ECM Cracow Conference Programme related to the ECA "IET SIG" is most warmly acknowledged.

\section{References}

[1] D. Vitkup, D. Ringe, G.A. Petsko, M. Karplus, Nat. Struct. Biol. 7, 34 (2000).

[2] A. Deacon, T. Gleichmann, A.J. Kalb (Gilboa), H. Price, J. Raftery, G. Bradbrook, J. Yariv, J.R. Helliwell, Faraday Trans. 93, 4305 (1997).

[3] J. Habash, J. Raftery, S. Weisgerber, A. Cassetta, M. Lehmann, P. Hoghoj, C. Wilkinson, J.W. Campbell, J.R. Helliwell, Faraday Trans. 93, 4313 (1997). 
[4] J. Habash, J. Raftery, R. Nuttall, H.J. Price, C. Wilkinson, A.J. Kalb (Gilboa), J.R. Helliwell, Acta Crystallogr. D 56, 541 (2000).

[5] H.J. Price, Ph.D. thesis, University of Manchester, 1999.

[6] E.H. Snell, N.E. Chayen, J.R. Helliwell, Biochemist 21, 19 (1999).

[7] A.J. Kalb (Gilboa), D. Myles, J. Habash, J. Raftery, J.R. Helliwell, J. Appl. Crystallogr. 34, 454 (2001).

[8] J.R. Helliwell, C.C. Wilson, in preparation.

[9] Report of the ESS Biology and Biotechnology Science Definition Group, 2001, Engelberg Workshop (copies available from john.helliwell@man.ac.uk). 